\title{
La Interpretación Como Evento Cognitivo EXPRESADo en Razonamientos AbDuCtivos
}

\author{
Maria G. Navarro ${ }^{1}$
}

RESUMEN: En esas páginas propongo una definición de la acción de interpretar según la cual esta actividad produce una forma específica de eventos denominados interpretaciones que preforman la actividad cognitiva de interpretar. Además, sostengo que en algunos casos la actividad de interpretar consiste en un proceso encaminado a la búsqueda de premisas plausibles para la formación de hipótesis explicativas consistentes por lo que la lógica de la interpretación puede ampliar el estudio de la llamada reproducción o generación de hipótesis.

PALABRAS CLAVE: Interpretación. Proposición. Abducción. Competencia inferencial. Sesgo cognitivo. Inferencia rebatible. Scott Soames.

\section{SCOTt SoAmes}

Se puede afirmar que las proposiciones son cruciales para cualquier teoría cognitiva. Las proposiciones tienen una vida más allá del lenguaje debido a que son en parte resultado del contenido de estados cognitivos. Además,

\footnotetext{
1 Instituto de Filosofía. Centro de Ciencias Humanas y Sociales (CSIC). María G. Navarro es licenciada en filosofía por la Universidad Complutense de Madrid. Doctora con mención europea en Filosofía por la UNED, Especialista Universitaria en Argumentación Jurídica por la Facultad de Derecho de la Universidad de Alicante y PhD candidate en CC. Políticas y Sociología. Ha realizado estancias de investigación en las Universidades de Heidelberg, TU-Berlín y Freiburg i/B, así como en el Instituto de Gestión de la Innovación y del Conocimiento del CSIC/UPV y en el Departamento de Inteligencia Artificial de la Facultad de Informática de la UCM. Obtuvo un contrato postdoctoral en la Universidad de Ámsterdam en convocatoria pública del Ministerio de Ciencia e Innovación/ Fullbright. Actualmente es contratada postdoctoral Juan de la Cierva en el CSIC. Sus áreas de interés académico son la hermenéutica filosófica, las teorías de la argumentación, la epistemología y la filosofía del lenguaje. Es autora del libro Interpretar y argumentar (Madrid/ México: Plaza y Valdés/ CSIC, 2009). Su último artículo es "On Fuzzines and Ordinary Reasoning", en: TRILLAS, Enric; SEISING, Rudolf; MORAGA, Claudio; TERMINI, Settimo (Ed.). On Fuzziness. A Homage to Lotfi A. Zadeh (Studies in Fuzziness and Soft Computing Vol. 216). Berlin, New York [et al.]: Springer, 2012.
} 
tienen la particularidad de satisfacer distintas funciones simultáneamente: la función lingüística, la perceptiva y la de representar contenidos cognitivos. Es debido a la posibilidad de satisfacer simultáneamente estas tres funciones por lo que Scott Soames entiende las proposiciones como minimal units of their representational type.

Soames $(2010 ; 2012)$ ha propuesto una definición de proposición según la cual esta sería entendida como un tipo de evento cognitivo. Las proposiciones son frecuentemente parte de grandes estructuras cognitivas que muestran en cierto modo el holismo y la interconexión contrafactual presente en los estados perceptivos. En What is Meaning? Soames (2010) sostiene una teoría sobre la proposición como un tipo de evento cognitivo basándose en dos ideas fundamentales: (1) que la actividad perceptiva y cognitiva de los agentes es la base conceptual de toda representación y (2) que las proposiciones son representacionales en virtud de las relaciones que mantienen con la actividad de representar. En estas páginas asumo la noción de proposición de Soames con la finalidad de definir el proceso mediante el cual interpretamos algo como algo.

Una proposición es un tipo de entidad que no es idéntica a la sentencia que la expresa puesto que la misma proposición puede ser expresa por sentencias distintas. Las proposiciones tendrían que ver con los contenidos de estados perceptivos y cognitivos. Sin embargo, la dificultad vuelve a aparecer en este punto. Porque al igual que en el caso de las sentencias, tampoco estos contenidos se pueden identificar sin más con las proposiciones. Con las proposiciones nos representamos el mundo como siendo de una determinada manera; pero precisamente es mediante las proposiciones como llegamos a representárnoslo de otro modo. A pesar de estas dificultades a la hora de determinar qué clase de entidad es una proposición, tradicionalmente se sigue insistiendo en que son en parte sentencias y en parte contenidos de nuestros estados perceptivos y cognitivos, y que consiguientemente son portadoras de verdad y falsedad.

La concepción de la proposición como tipo de evento cognitivo presenta a ésta como algo más que el mero efecto de casos en los cuales nos representamos cosas siendo de una u otra manera. Soames formula esta idea presentando el siguiente caso hipotético: La afirmación de que $h$ es rojo es un tipo de evento en el que un agente predica la rojez de $h$, ello representa $h$ como siendo rojo porque todos los casos concebibles acerca de ello son eventos en los cuales un agente lo hace así. Esto lleva a Soames a sostener que la forma mínima de conocimiento con las proposiciones radica en la capacidad de representar el 
mundo cognitiva o perceptualmente predicando propiedades de objetos, y así generar pruebas de tipos de eventos correspondientes a dichas predicaciones. Para obtener una forma más sólida de conocimiento uno debe ser capaz de construir proposiciones (y convertirlas en) objetivo de predicación.

Soames entiende la acción de predicar como un tipo de evento cognitivo, y esto implica que dicha acción es análoga al verbo intensional transitivo "buscar". Cuando una proposición es un tipo de evento para la predicación de la propiedad de un objeto $h, h$ puede ser constituyente de la proposición - en el sentido de que la proposición es definida en términos de $h$-, sin que la existencia de $h$ sea necesaria para la existencia de la proposición.

Tanto las proposiciones como las sentencias son tipos de eventos complejos que suponen la realización de ciertas acciones básicas. ¿Qué garantiza la existencia de proposiciones? La respuesta parece tan sencilla como radicalmente original: la existencia de eventos que preforman dichas acciones. Eso implica que muchas proposiciones no han sido ni serán nunca consideradas.

Las proposiciones se asumen y emplean como tipos de eventos en los cuales los agentes preforman ciertas operaciones cognitivas de representación. Tradicionalmente se entiende que una interpretación es el producto o resultado de la actividad de interpretar. Puede distinguirse la actividad de interpretar de sus productos, las interpretaciones. Las interpretaciones, entendidas como producto, se expresan en proposiciones. Siguiendo a Soames, se podría establecer un paralelismo entre lo que tradicionalmente se entiende por proposición y lo que comúnmente se entiende por interpretación. Proposición e interpretación son entidades que satisfacen B1-B3.

B1 Algunas cosas son interpretadas. Estas actitudes relacionan a aquellos que interpretan algo con aquello que interpretaron.

B2 Las cosas interpretadas son portadoras de verdad y falsedad.

B3 Las interpretaciones — aquello que satisface B1 y B2 - se expresan en sentencias. Las interpretaciones expresadas por $\mathrm{S}$ pueden ser designadas por las expresiones [la proposición de que S], [la declaración/afirmación/ creencia de que $S$ ], o simplemente [que $S$ ].

En esas páginas proponemos una definición de la acción de interpretar según la cual esta actividad produce una forma específica de eventos denominados 'interpretaciones' que preforman la actividad cognitiva de 
interpretar. Eso implica que, en efecto, muchas interpretaciones no han sido aún expresadas. Así como una proposición es una entidad que no es idéntica a la sentencia que la expresa - la misma proposición puede ser expresada por sentencias distintas - la actividad de interpretar es un tipo de evento cognitivo que no se puede identificar sin más con sus productos: las interpretaciones. Existen proposiciones que podrían presentarse finalmente como el producto de ese tipo de evento cognitivo (su interpretación resultante). $\mathrm{Ni}$ las proposiciones son idénticas a las sentencias en las que aquéllas se expresan (existen diferentes sentencias o expresiones que expresan la misma creencia), ni las interpretaciones se identifican con las sentencias.

La semántica necesita de las proposiciones para especificar los contenidos semánticos: qué es afirmado y creído por los hablantes. La misma suerte corren las interpretaciones desde el punto de vista semántico, a saber: son un tipo específico de entidad que representa el mundo como siendo de cierta manera; por esa razón se cree que para ellas existirían condiciones de verdad.

Cuando no se identifica con corrientes filosóficas como la hermenéutica o incluso con una visión continental de la actividad filosófica en contraposición a la analítica, el problema de la interpretación suele abordarse bien desde una perspectiva semántica, bien desde la pragmática (DASCAL, 1995; NAVARRO, 2009). En este sentido, un ejemplo paradigmático es el de Bunge (1974) quien distingue la interpretación como concepto semántico de la interpretación entendida en su referencia a los hechos sociales. Aunque no nos persuaden los argumentos empleados por Bunge para defender la separación entre la interpretación semántica y la interpretación como problema de las ciencias sociales, compartimos la idea según la cual la interpretación semántica se refiere a signos y constructos. Según esta acepción, el significado de un constructo no debe confundirse con el proceso mental de comprensión, es decir, una interpretación no debería confundirse con la actividad mental de interpretar.

Los productos de la actividad de interpretar no obtienen su carácter representacional de las sentencias en que aquéllas se expresan (KING, 2007), sino del hecho de que las interpretaciones (según sostenemos aquí) son el contenido de estados cognitivos intencionales.

¿Qué hecho convierte en legítima la pretensión de ir más allá de la acepción semántica y/o la pragmática de la actividad de interpretar? ¿Por qué la actividad de interpretar se define aquí como un tipo de evento cognitivo? Solemos elegir una interpretación que expresa una proposición $p$ cuando 
es parte del contenido de un estado perceptivo o cognitivo que deseamos caracterizar. Es por ello por lo que Soames afirma que utilizamos frases para expresar contenidos cognitivos complejos que representan otros contenidos cognitivos o perceptuales como satisfaciendo diversas condiciones. Como estamos viendo, este análisis de la actividad de interpretar no se limita a la acepción semántica ni a la pragmática.

Por esa razón, en estas páginas propongo analizar la actividad de interpretar como un tipo de evento cognitivo que adopta la forma de un razonamiento abductivo. Para dar un primer paso en esa dirección es necesario situar la actividad de interpretar no sólo en la tradición representacional sino en la inferencial.

\section{HaCIA UNA LÓgICA DE LA INTERPRETACióN}

Según Brandom (1994) la epistemología ilustrada fue el hogar de dos concepciones distintas acerca del origen, orden y conexión de las ideas. Por una parte, estaría la noción de representación defendida por Descartes quien al dividir el mundo en quienes por naturaleza son sujetos de conocimiento y efectúan representaciones (res cogitans), y aquello que es objeto de conocimiento y constituye una representación (res extensa), convertía el acto de poseer contenidos de representación en una instancia explicadora pero inexplicable.

A diferencia de Descartes, tanto Leibniz como Spinoza se preguntaron qué indica que una cosa represente a otra; pero lo hicieron en función de la significación inferencial de lo que representa. Esto último debía dilucidarse en todo caso a través de las relaciones inferenciales.

Como sabemos, también los empiristas británicos compartían el punto de vista según el cual las relaciones inferenciales se debían extraer de los contenidos de las representaciones, ya que la sola representación que un sujeto efectúe sobre algo no permite percatarnos al resto de los sujetos de que su representación verse en efecto sobre algo. Es entonces cuando se interpreta la corrección inferencial a partir de la corrección e inteligibilidad de la representación o representaciones. Desde entonces, en buena medida se ha tratado de definir las propiedades representadoras en función de las inferenciales.

En la historia de la filosofía de la lógica, la definición y análisis de los problemas sintácticos y los semánticos han sido constantemente relacionados 
con las propiedades representacionales y las inferenciales. Por ejemplo, suele decirse que los problemas sintácticos se relacionan con la inferencia válida, los conceptos de demostración y derivación, así como el método axiomático; mientras que los problemas semánticos se relacionarían con el análisis de los conceptos de significado y verdad, la distinción entre intensión y extensión, además de las paradojas y las antinomias.

El problema de la lógica de la interpretación no ocupa en la historia de la filosofía del lenguaje un puesto tan destacado como el de representación o el de inferencia. No obstante, la lógica de la interpretación está relacionada con estos dos últimos conceptos. En este artículo partimos del supuesto de que el problema de la interpretación se debería integrar en la formulación de problemas epistémicos asociados a la actividad cognoscitiva de la que se ocupan la tradición representacional y la inferencial.

Guardando una estrecha relación con la distinción entre los problemas sintácticos y los semánticos, la tradición aristotélica y medieval ya diferenció la logica utens de la logica docens (BEUCHOT, 2000). La hermenéutica se asemejaría consiguientemente a la lógica en tanto en cuanto corpus que puede ser, al unísono, arte y disposición de procedimientos susceptibles de aplicación en todos los razonamientos. Por ello, en la antigüedad se aceptaba que la hermenéutica se ocupaba de la aplicación de reglas y principios metodológicos o lógica utens, pero también del análisis de su contenido en tanto en cuanto teoría de la interpretación o lógica docens.

Siglos después, y debido a las separaciones teóricas acentuadas por la comprensión analítica de la filosofía frente a la tradición continental, los epistemólogos y filósofos del lenguaje se encuentran con que ni las meras representaciones ni nuestras concepciones acerca de la validez inferencial pueden explicar consistentemente los fenómenos asociados a la comprensión y al empleo de razonamientos.

Para todo proceso interpretativo hay un razonamiento abductivo que esclare el esquema lógico de dicho proceso. Para entender en qué momento interviene el uso del razonamiento abductivo en la actividad cognitiva de interpretar es necesario preguntarse: ¿qué significa interpretar?, o ¿qué hacemos cuando interpretamos? La interpretación es un fenómeno complejo formado por dos estadios plenamente integrados, si bien no son fácilmente distinguibles. En el primero, tras la efectuación de una comprensión, se formula una proposición en la que algo es tomado como algo. A continuación, 
se examina desde el punto de vista semántico la correlación y adecuación de aquello que se afirma en relación con un conjunto de proposiciones que forman parte de una teoría.

Postular la existencia de estos dos estadios en el fenómeno de la comprensión efectuada, esto es, en el de la interpretación propiamente dicha, conlleva que si aceptamos que interpretar implica tomar algo como algo o en tanto que algo, entonces, ninguna proposición podría encerrar, en sí misma, las condiciones mediante las cuales habríamos de atisbar su comprensibilidad, puesto que la efectuación de dicha comprensión no podría idenficarse, de hecho, con ninguna forma de totalidad. Es a esto a lo que se ha referido la tradición de la hermenéutica filosófica con la idea de que la conciencia histórica desborda su propio conocimiento de sí.

La inferencia abductiva también denominada inferencia de la mejor explicación es una forma de razonamiento que parte de un conjunto de datos para concluir una hipótesis que explica plausiblemente ese conjunto de datos. Todos los autores que se han dedicado a su estudio comparten la opinión de que es un tipo de inferencia especial, de naturaleza tentativa y, por ello, capaz de generar nuevos conocimientos. A pesar de ello, no han sido suficientemente investigadas las conexiones entre la inferencia abductiva y disciplinas como la psicología cognitiva y, en particular, la psicología de la inducción, además de la sociología del razonamiento, la argumentación visual e incluso la investigación arqueológica.

Uno de los fines de este tipo de razonamiento consiste en representar, en sí mismo, un método para la adquisición de conocimiento, esto es, un modelo de inferencia ampliativo. El modelo de razonamiento abductivo es especialmente creativo y flexible a la hora de encontrar por ejemplo aplicaciones a prácticas concretas como el diagnóstico médico, los procesos de comprensión de grandes conjuntos de datos, o cuando de lo que se trata es de diseñar heurísticas, producir conjeturas, hipótesis e incluso condicionales contrafácticos en áreas de conocimiento como la investigación histórica, y en general en cualquier otra actividad que exija un modelo de razonamiento en el que se interrelacionen los aspectos interpretativos, creativos y lógicos para la producción de hipótesis de sentido.

Si admitimos que cabe describir así el fenómeno que tiene lugar cuando interpretamos, entonces, podemos afirmar que la actividad de interpretar exige hacer uso de razonamientos tentativos e incluso probabilísticos. La 
interpretación es un tipo de evento cognitivo en el que puede apreciarse el esquema de una inducción abductiva.

Las concepciones acerca de la validez inferencial no pueden explicar por sí solas los fenómenos de comprensión y razonamiento debido a las dificultades teóricas implícitas en estos tres temas: la noción de competencia inferencial, el concepto de sesgo cognitivo y, por último, las llamadas inferencias rebatibles.

Los resultados de la investigación experimental llevada a cabo por Wason (1960) son un ejemplo paradigmático de cómo las concepciones acerca de la validez inferencial no dan cuenta satisfactoriamente de los fenómenos de razonamiento ordinario.

Fue en 1960 cuando algunos psicólogos experimentales (WASON, 1966) empezaron a interesarse por la naturaleza del razonamiento humano llevando a cabo una serie de experimentos que les permitían concluir que la mayoría de los seres humanos comenten ordinariamente errores básicos de orden deductivo en sus inferencias. Como resultado de esta investigación surge el concepto de competencia inferencial cuya definición no se adapta a los principios y reglas de la lógica estandarizada ${ }^{2}$.

Esta investigación se enriqueció más adelante con la aplicación de una hipótesis tentativa según la cual los sujetos de los experimentos realizaban inferencias más consistentes desde el punto de vista formal cuando se les encarga la resolución de dichas tareas utilizando el lenguaje natural, y no a través de formalizaciones en un lenguaje objeto.

La investigación de aquellos psicólogos experimentales está en sintonía con el descubrimiento de sesgos cognitivos, es decir, errores sistemáticos a la hora de realizar inferencias. Desde el punto de vista de la psicología cognitiva, los sesgos cognitivos son uno de los conceptos nucleares de la psicología del razonamiento (BISHOP; TROUT, 2005). Habría cierto tipo de contextos, condiciones y situaciones en las cuales un mecanismo cognitivo (con efectos inferenciales o inductivos) produce resultados cognitivos que no son correctos. Este descubrimiento llevó a Gigerenzer (1991) a considerar la existencia de una cierta heurística cognitiva. Este concepto nos lleva aquí a presentar la hipótesis según la cual en el razonamiento ordinario los procesos inferenciales y representacionales se abordan a partir de procesos interpretativos. En el

\footnotetext{
${ }^{2}$ La lógica clásica reconoce solamente dos valores de verdad a sus fórmulas (verdadero o falso), por lo que también se la denomina lógica bivalente o lógica estandarizada, e incluye a la lógica proposicional, la lógica de predicados, la lógica de clases y la lógica de relaciones.
} 
caso de los sesgos epistémicos, es la previa interpretación de los contextos en los cuales se llevan a efecto mecanismos cognoscitivos (una inferencia, una representación) lo que orienta al sujeto a la hora de producir mecanismos cognoscitivos justificados o aceptables (GARCÍA AGUILAR, 2004).

Por todo ello, autores como Steninng y Lambalguen (2001) han llamado la atención en sus estudios acerca de las inferencias rebatibles (defeasible inferences) sobre la poca importancia que suele concedérsele a la interpretación en el dominio de la lógica y la filosofía del lenguaje. Estos autores han analizado las causas relacionadas con las dificultades de razonamiento que presentan algunos autistas a la hora de realizar inferencias a partir de la información recibida presumiblemente por no realizar implicaturas conversacionales, es decir, por prestar más atención a las normas de inferencia propiamente dichas que a las máximas conversacionales.

Las inferencias rebatibles son lo opuesto de los argumentos deductivos los cuales no son rebatibles, es decir, si una conclusión se sigue deductivamente de un conjunto de premisas $p$, nunca puede ser válida si se aumenta $p$, o sea, una inferencia válida no puede ser válida si, entre otros aspectos, se obtiene más información a partir de ella.

Sin embargo, en la vida cotidiana nuestras inferencias no son así. Lo podemos comprobar, por ejemplo, a través de algunas de las más importantes inferencias revocables o rebatibles tales como las inferencias condicionales, dentro de las cuales cabría destacar —entre otras - las llamadas implicaturas conversacionales. Como recordamos, Grice (1975) defiende la existencia de una serie de normas o máximas conversacionales - conocidas tanto por el hablante como por el oyente-que guían la conversación y hacen posible que las inferencias deducidas por el oyente sean exactamente las que el hablante había querido comunicar. A este tipo de inferencias que el oyente deduce y que no dependen de las palabras sino de las máximas conversacionales, Grice las llama implicaturas conversacionales. Tanto las implicaturas conversacionales como las abducciones forman parte del modelo condicional de inferencia.

Con el propósito de presentar nuestra visión del razonamiento abductivo entendido como patrón lógico presente en los procesos interpretativos, en lo que sigue, abordaremos algunas de las más perspectivas más destacadas dando lugar a una historia comprensiva, y por ello tal vez limitada (comparada con los estudios lógicos de Kakas, Kowalski, et al, 1987; Poole, Goebel, et al, 
1992) de la representación del patrón abductivo (más extensamente elaborado en NAVARRO, 2010; NAVARRO y DE HARO, 2012).

\section{Aproximaciones al patrón LóGico ABDUCTIVo}

Suele decirse que los dos modos básicos de razonamiento son la deducción (inferencia desde las causas a los efectos o desde lo universal hacia lo particular) y la inducción (que recorrería el camino inverso). Sin embargo, la abducción representa un modelo de inferencia que no es deductiva ni inductiva por lo que constituye un tercer modelo de razonamiento al que se ha relacionado con la lógica inventiva o del descubrimiento científico más que con la de la justificación. Fue el filósofo norteamericano Charles $S$. Peirce quien desarrolló este tercer modelo de inferencia. Algunos autores han insistido en que es el primer modelo de inferencia puesto que genera nuevas ideas al consistir en el razonamiento mediante el cual se engendran hipótesis explicativas.

Según Murphey (1993) fue durante una conferencia en el Lowell Institute donde Peirce reveló cómo había hallado los tres modelos de inferencia. $\mathrm{Al}$ parecer, durante la lectura del tratado de George Boole, Laws of Thought, Peirce se planteó qué es la inducción a la luz de las investigaciones de Boole sobre la probabilidad.

Es importante señalar que el hallazgo de Peirce se encuentra ya desarrollado en los capítulos 23, 24 y 25 del libro Segundo de los Primeros Analíticos de Aristóteles donde este denomina epagoge al procedimiento por medio del cual se infiere la premisa mayor de un silogismo a partir de la premisa menor y de la conclusión ${ }^{3}$. Este tipo de inferencia no es reducible a una deducción necesaria, y supone un modo de argumentar independiente de la deducción.

Algunos autores prefieren referirse al proceso abductivo mediante la expresión inference to the best explanation (en adelante IBE) o inferencia de la mejor explicación. Tal vez esta expresión sea más intuitiva pero aquí utilizaremos las dos pues la literatura existente mantiene los dos términos. A pesar de ello, conviene recordar que en el caso de autores como Woods y Gabbay (1995) no está claro que abducción e IBE se puedan utilizar como sinónimos. La diferencia fundamental radica en que con la abducción

${ }^{3}$ El término epagoge suele traducirse por comprobación y, a veces, por inducción. 
nos referimos al proceso por medio del cual se obtienen diversas hipótesis explicativas. Sin embargo, con la IBE se plantea el problema secundario de pensar en los criterios que deberían aplicarse para determinar cuál de aquellas hipótesis es la respuesta correcta o la explicación verdadera. En este sentido, la abducción únicamente modela el cambio de creencias, o sea, describe un proceso cognitivo (ALISEDA, 2006).

Más adelante, en el famoso artículo Deducción, inducción e hipótesis, Peirce (1878) simplifica sus observaciones sobre los tres modos de inferencia a partir de la siguiente explicación: el silogismo categórico no es más que la aplicación de una regla a un caso para establecer un resultado (es analítico o explicativo). Sin embargo, el razonamiento abductivo supone la inferencia de un caso a partir de una regla general y un resultado. Este tipo de inferencia es, por ese motivo, conjetural e hipotética.

Peirce $(1965,5.145)$ también se refiere al argumento abductivo utilizando esta fórmula:

El hecho sorprendente, $\mathrm{C}$, es observado

Pero si A fuera verdadera, se daría C

Luego hay razón para sospechar que A es verdadera

Esta última es la fórmula que Peirce llamó hipótesis o, mejor dicho, expresión hipotética de la abducción; y es la formulación que más suele citarse. Será a partir de los trabajos de Harman (1965) cuando la expresión hipotética de la abducción pase a ser conocida como inferencia de la mejor explicación. Pues bien, es al hilo de esta disquisición cuando Peirce se pregunta qué diferencia existe entre una inferencia y una hipótesis. Concluye que la gran diferencia entre la inducción y la hipótesis estriba en que la primera infiere la existencia de fenómenos iguales a los que hemos observado en casos similares, mientras que la hipótesis supone algo distinto a lo que hemos observado directamente.

Peirce sostiene que al menos existen tres tipos de hipótesis:

i. acerca de entidades o hechos no observados en el momento de formular la hipótesis pero observables en el futuro para verificarla;

ii. acerca de entidades o hechos que alguien pudo observar aunque no sea posible repetir la observación por ser hechos del pasado;

iii. acerca de entidades inobservables en la práctica. 
Teniendo a la vista que es a la luz de la producción de hipótesis como cabe entender este tipo de razonamiento, una manera más sencilla de presentar el patrón en el que se basa la inferencia de la mejor explicación puede ser este que sigue:
$\mathrm{D}$ es un conjunto de datos pendiente de explicación y/o comprensión
$\mathrm{H}$ es una hipótesis que de ser verdadera haría explicable y/o comprensible $\mathrm{D}$
$\mathrm{H}$ es una conjetura plausible

Como podemos ver, la abducción tiene que ver con la formación de hipótesis, es decir, responde a la pregunta acerca de cómo llegar a conjeturas a partir de las cuales dar con una interpretación cabal de un conjunto de datos. Por consiguiente, no es una inferencia probable sino una especie de suposición que se adopta provisionalmente.

Según Thagard (1988, p. 52) existen diversos tipos de abducción, y cada una de ellas juega un papel distinto en las llamadas estrategias heurísticas que intervienen en los descubrimientos científicos. Para Thagard existirían cuatro tipos de abducción:

i. la abducción simple [modo de obtener hipótesis explicativas a partir de la observación de un hecho: Todo $\mathrm{S}$ es $\mathrm{P}$; a es $\mathrm{P}$, tenemos razones hipotéticas para afirmar que "a es $S$ "];

ii. la abducción existencial [la hipótesis obtenida es una afirmación de carácter existencial];

iii. la abducción para la formación de reglas [se plantea a veces como una forma de generalización inductiva];

iv. la abducción analógica [aplica la analogía para la formulación de hipótesis que explican otros fenómenos conocidos: dado el hecho $\mathrm{C}_{1}$ que viene perfectamente explicado en la hipótesis $\mathrm{H}$, dado que el hecho $\mathrm{C}_{2}$ fue análogo a $\mathrm{C}_{1}$ se abduce $\mathrm{H}$ como una explicación de $\mathrm{C}_{2}$ ].

Para todos los casos se mantiene que a partir de hechos conocidos pueden ser abducidas más de una hipótesis. Esto es lo mismo que afirmar que, en muchas ocasiones, para un mismo hecho nos encontramos con la existencia de múltiples interpretaciones satisfactorias. Este fenómeno conduce a la representación de la abducción en la que ésta pasa a ser entendida como inferencia de una explicación potencial y, en este sentido, como la inferencia de la mejor explicación (NIINILUOTO, 1999). Es a la luz de esta definición 
de abducción como cabe entender el importante estudio de J. Josephson (1994) en el que se describe el patrón abductivo de la siguiente manera:

$\mathrm{D}$ es una colección de datos

$\mathrm{H}$ explica D (si fuera verdadera, explicaría D)

Ninguna otra hipótesis puede explicar $\mathrm{D}$ tan bien como lo hace $\mathrm{H}$

Por tanto, $\mathrm{H}$ es probablemente verdadera

Parece razonable que Hintikka (1999) llegara a afirmar que la abducción es el problema fundamental de la epistemología contemporánea. Según Hintikka las inferencias abductivas son respuestas a preguntas del investigador, en base a ello propone la siguiente caracterización de la abducción:

i. la abducción constituye un proceso inferencial;

ii. los objetivos epistémicos propios de la abducción se pueden resumir en dos: generar nuevas hipótesis en el curso de una investigación; seleccionar hipótesis para su posterior verificación;

iii. el modelo de razonamiento abductivo está presente en la producción de todas las teorías científicas;

iv. la abducción es un proceso inferencial irreducible a otras formas de inferencia.

Algunos autores (RIVADULLA, 2004) han llegado incluso a plantearse si es posible un enfoque bayesiano de la inferencia abductiva. El problema de la inferencia bayesiana reside en que si tras una acumulación de evidencia favorable a una hipótesis, una nueva evidencia la contradijese, esa hipótesis probablemente verdadera quedaría refutada. Por lo tanto, Rivadulla sostiene que hay que desterrar la pregunta acerca de qué probabilidad de ser verdadera tiene una determinada teoría científica porque no prescribe condiciones de selección de predicciones ni su secuencia temporal. En este sentido rechaza el enfoque bayesiano de la abducción.

Más recientemente, el historiador Peter Lipton (2004) afirma que la IBE invierte el punto de vista común sobre la relación entre la inferencia y la explicación. Según el punto de vista natural o del sentido común, la inferencia sería anterior a la explicación. Sin embargo, el modelo de razonamiento implícito en la IBE consiste en analizar comparativamente hasta qué punto consigue explicar la evidencia en cuestión un conjunto de hipótesis. De modo que en este modelo, la IBE (y por tanto la explicación) es, por así decir, anterior a la inferencia. 
En lo que sigue describiré brevemente algunas de las aplicaciones más destacadas del proceso de razonamiento abductivo. Es posible que la gran variedad de aplicaciones de este tipo de razonamiento se deba al hecho de que la actividad de interpretar — presente, según afirmamos aquí, en dicho proceso- es un tipo de evento cognitivo que no se puede identificar con sus productos: las interpretaciones.

\section{Aplicaciones DEL RAZONAMIENTo abDUCTIVo}

la utilización que se ha hecho de este modelo de razonamiento para dar cuenta de los procesos cognoscitivos que intervienen en el hallazgo de descubrimientos científicos es excesiva si tenemos presente que el hallazgo científico y las lógicas de la invención no son exclusivas de las ciencias experimentales. Con todo, es además sesgada en sí misma ya que lo que lleva al hallazgo o descubrimiento son los componentes interpretativos propios de la argumentación tópica presentes en los procesos cognitivos de naturaleza representacional e inferencial.

Por ejemplo, en el ámbito de la arqueología, Shelley (1996) ha puesto de relieve el importante rol que juega la representación de imágenes visuales en la construcción de nuevas hipótesis. Y es que, en el caso de la arqueología donde el descubrimiento de algunos objetos puede tomarse como indicio de la existencia de otros que no están presentes, es notoria la necesidad de utilizar razonamientos abductivos para descubrir restos o conjuntos arqueológicos.

En esta disciplina el empleo del razonamiento abductivo está relacionado con el descubrimiento de formas cuando no de restos materiales que podrían adquirir (bajo ciertos supuestos) ciertas formas o figuras; y por ello ha dado lugar a las siguientes distinciones conceptuales:
i. la abducción relacionada con la forma de los objetos;
ii. la abducción relacionada con la estructura de los objetos;
iii. la abducción que produce inferencias analógicas.

De este ejemplo podemos extraer dos ideas de interés, a saber, que el razonamiento abductivo está relacionado con un proceso en el que se presentan ciertos signos (eventualmente interpretados) como detonadores abductivos (ALISEDA, 2006). Y en segundo lugar, que los detonadores abductivos 
aludirían a dos fenómenos concretos: el de novedad (un fenómeno novedoso) y el de anomalía (hallarse ante una anomalía).

Aunque no se haya señalado nunca anteriormente, cabe decir que esta descripción del fenómeno abductivo está implícita en los estudios de Gorleé (1996) en torno al uso de la abducción en la traducción interlingüística. Su aplicación se hace patente en los casos de la traducción descriptiva que se concentran en la traducción como producto. Ese es el caso de la traducción entendida como transferencia en la que se reconoce que existen similitudes que justifican la traducción que se da como válida pero de modo transitorio o derivado de un texto porque se dé el caso de que éste pertenezca, por ejemplo, a una cultura diferente. En este sentido las hipótesis explicativas utilizadas en pasos anteriores afectan a los procedimientos de investigación e interpretación ulteriores.

Por este motivo, algunos autores (TURSMAN, 1987) consideran que el uso de procesos abductivos en este tipo de estudios quedaría descrito con la metáfora explicativa de la figura helicoidal más que con la lineal, ya que siempre quedaría algo hacia lo que volver, algo que de alguna manera podría volver a ser descubierto. El método lógico-semiótico de Peirce sería por ello totalmente aplicable a la identificación, descripción y análisis de la traducción como un experimento mental de generación de significado en el que una hipótesis producida abductivamente es comprobada de modo reiterado.

En cuanto a la aplicación del razonamiento abductivo en Inteligencia Artificial (IA), según Aliseda (2006) ésta se remonta a los años setenta aunque es en la década de los noventa cuando se aplica en la programación lógica, el reconocimiento de patrones, el procesamiento de lenguaje natural y, en general, como modelo de razonamiento de carácter revocable. A su vez, la aplicación de la IA al ámbito del diagnóstico médico, ha devuelto este modelo de razonamiento a sus orígenes como método indiciario. Por ejemplo, la aplicación del modelo del diagnóstico abductivo en una unidad de cuidados intensivos ha dado lugar a nuevas aplicaciones y concepciones de la abducción.

Sin embargo, la gestión de información temporal e interacción inteligente en medicina va más allá de los métodos convencionales de razonamiento abductivo. Según los métodos convencionales, primero se suele procesar la etapa abductiva para generar una explicación que cubra todas las manifestaciones. A continuación, se pasa por una etapa de diferenciación en la que la explicación obtenida en la etapa abductiva es refinada mediante la eliminación de algunas de las hipótesis. Sin embargo, el modelo que proponen 
algunos autores (POOLE et al., 1987) se adapta mucho más a los casos en los que se realizan operaciones constantes. Casos en los que además la explicación obtenida en un ciclo anterior de diagnóstico se refina eliminando hipótesis inconsistentes a la luz de nuevas manifestaciones. Para ello es necesario orientar la observación del proceso a través de parámetros discriminantes, los cuales determinan frecuentemente cuáles son las manifestaciones que pueden implicar modificaciones. Eso implica una mejora final a la hora de evaluar o reevaluar el proceso de interpretación.

Tras este periodo de diferenciación, existe una fase abductiva en la que se intenta extender la explicación para cubrir todas las manifestaciones anormales que se han producido tras el último diagnóstico. Y esto, a su vez, determina el conjunto de parámetros discriminantes para las nuevas hipótesis ańadidas, el cual se utilizará en la siguiente fase de diferenciación. En definitiva, se intenta diseñar un modelo con el que tener en cuenta aquellas hipótesis consistentes con la información obtenida hasta dicho instante ya que las hipótesis inconsistentes de cara a la nueva manifestación son eliminadas de la fase de diferenciación. La abducción estaría modelando por tanto la competencia cognitiva de los médicos.

Puede decirse que, en cierto modo, esta última aplicación de la abducción en el diagnóstico médico no hace sino conducir a la práctica la investigación básica presente en las investigaciones filosóficas y lógicas actuales acerca de la abducción. Estas últimas (JOSEPHSON; JOSEPHSON, 1996; THAGARD, 1978) tienden a considerar que las condiciones de consistencia e inconsistencia son internas a la abducción misma, por lo que la inconsistencia sería esencial a la abducción. Y lo cierto es que parece de sentido común que así sea porque si las hipótesis plausibles no fueran incompatibles entre sí, no existirían alternativas para explicar o interpretar. En cualquier caso, aquí es importante resaltar que en el mundo de la investigación aplicada al diagnóstico médico, se ha establecido una rica clasificación acerca de la naturaleza de las hipótesis. Un hecho que ya advirtieron Josephson y Josephson (1996) cuando concentraron su análisis en las máquinas abductivas.

Recapitulando, el estudio de la abducción nos hace advertir (entre otras cosas) que habría hipótesis compatibles, hipótesis que forman parte de un subconjunto de otra hipótesis, hipótesis que sugieren o implican el uso de otra(s), hipótesis excluyentes, etc. Esto último es sobresaliente desde un punto de vista filosófico e incluso historiográfico si se me apura, ya que las metodologías y aplicaciones son más complejas en la medida en que no sólo 
consideren únicamente hipótesis consistentes sino también las mutuamente excluyentes. Pero es que incluso para los estudios en torno a la abducción de esta última afirmación se puede extraer una conclusión: como resultado de asumir esa categorización y empleo de las hipótesis inconsistentes, la propia abducción se convierte - en relación a ellas - en una metodología para abordar la inconsistencia en cuanto tal (PSILLOS, 2000).

Tal vez sea el problema de pensar la inconsistencia y su presencia en contextos sociales concretos, lo que ha llevado a Patokorpi y Ahvenainen (2009) a afirmar que la abducción también podría utilizarse para el diseño social de soluciones tecnológicas para perspectivas futuras en los casos en los que bien la tecnología existente, bien la sociedad en un sentido amplio no consiguen explicar un dato o una experiencia que se nos presenta como inexplicable, sorprendente o inconsistente dentro de un marco teórico dado. Lo que proponen Patokorpi y Ahvenainen está en sintonía con una de las contribuciones más destacadas dentro de los estudios en abducción, a saber, la del filósofo y científico cognitivo Lorenzo Magnani quien recientemente ha descrito la abducción como un tipo de inferencia hipotética que realizan los seres humanos pero también los animales a través del pensamiento pero que se opera o materializa a través de acciones con las que, como resultado de alterar entornos físicos y culturales, se generan nuevos nichos cognitivos para el futuro.

Lo visto hasta ahora basta para hacerse una idea de las definiciones de abducción y sus aplicaciones, así como para reconocer la necesidad de definir conceptos relacionados con la abducción tales como: hipótesis, detonadores abductivos, anomalía abductiva, nicho cognitivo, affordance, etc.

\section{Conclusión}

La definición de proposición propuesta por Soames según la cual ésta es un tipo de evento cognitivo nos ha llevado, en primer lugar, a identificar la actividad de interpretar con la de producir tales eventos de la cognición; $y$, en segundo lugar, a presentar la tesis de que dicho evento constituye un proceso de razonamiento modelado según un patrón abductivo. Finalmente, esta propuesta tiene como consecuencia que presentar dicha definición de interpretación supone introducir la definición de proposición de Soames dentro de un problema filosófico seguramente no más amplio pero sí distinto: el de la hermenéutica filosófica. Seguramente el propio Soames es consciente de 
que hace radicar su propuesta en ese espacio fronterizo (entre lo analítico y lo continental) cuando afirma que la relación estructural entre los componentes de una proposición se deben a los roles que aquéllos mantienen en la secuencia de operaciones cognitivas realizadas por el agente que las utiliza (Soames, 2012). Roles como ser/estar siendo predicado (de ciertas cosas), ser objeto (de ciertas predicaciones), ser aplicado (a ciertos argumentos), ser argumentos (a los que se aplican ciertas cosas) no parecen poder entenderse sin suponer la actividad de interpretar.

Después de analizar someramente las contribuciones más notables al estudio de la abducción, se hace patente que en dicha tradición suele ser más un presupuesto que una tesis a desarrollar el hecho de que producir razonamientos abductivos sería de todo punto impensable si no partiésemos de una teoría o interpretación de fondo. Por esta razón, creo necesario volver a revisar el patrón inferencial de la abducción en sus distintas formulaciones con objeto de presentar su definición a la luz de ese marco primordial que es la interpretación de fondo o T[eoría]:

$$
\begin{aligned}
& \mathrm{T}=\text { una teoría acerca de fenómenos } \\
& \mathrm{O}=\text { observación de un fenómeno novedoso o extraño } \\
& \mathrm{H}=\text { hipótesis abducida a partir de } \mathrm{T} \text { y } \mathrm{O}, \text { y porque explica } \mathrm{O}
\end{aligned}
$$

Según mi punto de vista, la producción de teorías es un proceso intrínsecamente interpretativo (concebir $\mathrm{T}$; transformar $\mathrm{T}$ en $\mathrm{T}_{1}$; ampliar $\mathrm{T}_{1}$; rechazar $\mathrm{T}_{1}$ en virtud de $\mathrm{X}$, por tanto, producir $\mathrm{T}_{\mathrm{n} . . .}$ ). Los efectos ampliativos que observamos en la inferencia de la mejor explicación son el resultado de aplicar un modelo de razonamiento que va más allá de la lógica clásica por la sencilla razón de que factores como la adquisición de una lengua o la historicidad de nuestra comprensión preforman nuestras cogniciones tanto individual como colectivamente.

El patrón inferencial abductivo subyace en la interpretación si entendemos ésta como un tipo de evento cognitivo utilizado, por ejemplo, en la producción de nuevas interpretaciones o incluso en la producción de las principales hipótesis para el desarrollo de nuevas teorías. Por tanto, la producción de teorías constituye un proceso intrínsecamente interpretativo (concebir T; transformar $\mathrm{T}$ en $\mathrm{T}_{1}$; ampliar $\mathrm{T}_{1}$; rechazar $\mathrm{T}_{1}$ en virtud de $\mathrm{X}$, por tanto, producir $\mathrm{T}_{\mathrm{n} . . .}$ ). La adquisición de una lengua y la historicidad de nuestra comprensión preforman nuestra cognición a través del tiempo individual y colectivo. 
Por esta razón, los efectos ampliativos observados en la inferencia de la mejor explicación son el resultado de la aplicación de un modelo de razonamiento integrado en la acción de interpretar. Los parámetros inferenciales que determinan la relación lógica entre explandum y explanans, así como la explicación abductiva son inseparables de la competencia abductiva entendida en un sentido tanto teórico como manipulativo (MAGNANI, 2009). Vivir interpretando parece ser el fenómeno implícito en ese modelo y patrón de razonamiento; pero lo cierto es que sólo abundando en él podremos descubrir la complejidad inherente a ese modo fundamental de estar en el mundo. Que la interpretación se defina aquí como un tipo de evento cognitivo plasmado abductivamente implica que ese modo de estar en el mundo debe investigarse a partir de lo que en él hay de razonamiento en todos los seres vivos. Tal y como afirmaba Heidegger en Ser y tiempo no sólo la interpretación en general, también la proposición tiene necesariamente sus fundamentos existenciarios en el tener, el ver y el concebir previos.

\section{Agradecimientos}

El trabajo de investigación que ha dado como resultado este artículo forma parte del proyecto "Filosofía de la historia y valores en la Europa del siglo XXI” [FFI2008-04279/FISO] financiado por el Ministerio de Ciencia e Innovación español y del proyecto europeo ENGLOBE Marie Curie Initial Training Network [FP7-PEOPLE-2007-1-1-ITN] financiado por el Séptimo Programa Marco de la Comisión Europea.

NAVARRO, María G. Interpretations as cognitive event types expressed through abductive reasoning. Trans/Form/Ação, Marília, v. 35, n. 3, p. 231-252, Set./Dez., 2012.

\footnotetext{
ABSTRACT: The purpose of this paper is to argue that abductive reasoning is part of a cognitive process of interpreting something as being something. The paper makes two fundamental claims. First, it proposes a definition of interpretation as a type of cognitive event. Second, it is argued that in some cases the activity of interpreting is a process of searching for plausible assumptions for the consistent formation of explanatory hypotheses. As a result of these two theses, it is concluded that the logic of interpretation can enrich and extend the study of so-called reproduction or generation of hypotheses.
} 
The treatment given here to abduction is contrasted and integrated into the history of studies on logical patterns of abduction and their applications.

KEYWORDS: Interpretation. Proposition. Abduction. Inferential competence. Cognitive bias. Defeasible inferences. Scott Soames.

\section{REFERÊNCIAS}

ALISEDA, Atocha. Abductive Reasoning. Logical Investigations into Discovery and Explanation. Dordrecht: Springer, 2006.

ARRIGHI, Claudia; FERRARIO, Roberta. Abductive Reasoning, Interpretation and Collaborative Processes. Foundations of Science, v. 13, p. 75-87, 2008.

BEUCHOT, Mauricio. Tratado de hermenéutica analógica. Hacia un nuevo modelo de interpretación. México, D.F.: Ítaca, 2000.

BISHOP, Michael A.; TROUT, J. D. Epistemology and the Psychology of Human Judgment. Oxford: Oxford University Press, 2005.

BRANDOM, Robert. Making It Explicit: Reasoning, Representing, and Discursive Commitment. Cambridge: Harvard University Press, 1994.

BUNGE, Mario. Treatise on Basic Philosophy. Vol. 2: Semantics II: Interpretation and Truth. Dordrecht, Holland: Reidel Publishing Company, 1974.

DASCAL, Marcelo. Epistemología, controversias y pragmática. Isegoría. Revista de filosofía moral y politica, 12, p. 8-43, 1995.

DOUVEN, Igor. Abduction. The Stanford Encyclopedia of Philosophy (Spring 2011 Edition). In: ZALTA, Edward N. (Ed.) URL = <http://plato.stanford.edu/archives/ spr2011/entries/abduction/>

GABBAY, Dov M.; WOODS, John. A Practical Logic of Cognitive Systems: The Research of Abduction. New York: Elsevier Science, 2005.

GARCÍA AGUILAR, Claudia Lorena. Especificidad de dominio y sesgo en la psicología de razonamiento humano. Signos Filosóficos, v. 6, no 12, p. 63-91, 2004.

GIGERENZER, Gerd. Why Heuristics Work. Perspectives on Psychological Science. v. 3, no 1, p. 20-29, 1991.

GORLEÉ, Dinda L. A Eureka Procedure: Pragmatic Discovery in Translation. European Journal for Semiotic Studies, v. 8 (2, 3), p. 241-269, 1996.

GORLEÉ, Dinda L. On Translating Signs: Exploring Text and Semio-Translation. Amsterdam and New York: Rodopi, 2004.

GRICE, Paul. Logic and Conversation. In: COLE, Peter; MORGAN, Jerry L. (Ed.). Syntax and Semantics. New York: Academic Press, 1975, p. 41-45. 
HARMAN, Gilbert H. Inference to the best explanation. The Philosophical Review, v. 74, no 1 , p. 88-95, 1965.

HELMREICH, Stefan. Induction, deduction, abduction, and the logias of race and kinship. American Ethnologist, v. 34, no 2, p. 230-232, 2007.

HINTIKKA, Jaakko. What is abduction? The fundamental problem of contemporary epistemology. Transactions of the Charles S. Peirce Society, v. 34, p. 503-533, 1999.

JOSEPHSON, John; JOSEPHSON, Susan. (Ed.) Abductive inference. Computation, Philosophy, Technology. Cambridge: Cambridge University Press, 1994.

JOSEPHSON, John; JOSEPHSON, Susan. Abductive Inference. Cambridge: University Press, 1996.

KAKAS, Antonis. C.; KOWALSKI, R. A.; TONI, F.: Abductive Logic Programming. Journal of Logic and Computation, v. 2, n'. 6, p. 719-770, 1992.

KING, Jeffrey C. The Nature and Structure of Content. Oxford: Oxford University Press, 2007.

LIPTON, Peter. Inference to the best Explanation. London: Routledge, 2004.

MAGNANI, Lorenzo. Model-Based and Manipulative Abduction in Science. Foundation of Science, v. 9, p. 219-247, 2004.

MAGNANI, Lorenzo. Abducing chances in hybrid humans as decision makers. Information Sciences, v. 179, p. 1628-1638, 2009.

MAGNANI, Lorenzo. Abductive Cognition. The Epistemological and Eco-Cognitive Dimensions of Hypothetical Reasoning. Berlin/ Heidelberg: Springer, 2009.

MAGNANI, Lorenzo; PIAZZA, Matteo. Morphodynamical Abduction. Causation by Attractors Dynamics of Explanatory hypotheses in Science. Foundations of Science, v. 10, p. 107-132, 2005.

MURPHEY, Murray G. The Development of Peirce's Philosophy. Indianapolis: Hackett, 1993.

NAVARRO, María G. Interpretar y argumentar. Madrid/México: CSIC/Plaza y Valdés, 2009.

NAVARRO, María G. Intelligent Environments and the Challenge of Inferential Processes. Tijdschrift voor Filosofie, v. 72(2), p. 309-326, 2010.

NAVARRO, María G.; DE HARO GARCÍA, Noemi. Cognitive Abduction and Visual Culture. In MAGNANI, Lorenzo; PING, Li. (Ed.). Philosophy and Cognitive Science, Western and Eastern Studies. Heidelberg/Berlin: Springer, Sapere, vol. 2, p. 205-220.

NIINILUOTO, Ikka. Defending Abduction, Philosophy of Science, v. 66, no 3, p. 436S451, 1999.

PATOKORPI, Erkki; AHVENAINEN, Marko. Developing an abduction-based method for futures research. Futures, v. 41, p. 126-139, 2009. 
PEIRCE, Charles Sanders. Collected Papers. Cambridge, Mass.: Harvard University Press, 1965 [5.145].

PEIRCE, Charles Sanders. Deducción, inducción e hipótesis. Buenos Aires: Aguilar, 1970, p. 65-90 [W3. 323-338, 1978].

POOLE, David; GOEBEL, Randy; ALELIUNAS, Romas. Theorist: A Logical Reasoning System for Defaults and Diagnosis. In: CERCONE N.J.; MCCALLA, G. (Ed.). The Knowledge Frontier: Essays in the Representation of Knowledge. New York: SpringerVerlag, p. 331-352, 1987.

PSILLOS, Stathis. Abduction: between Conceptual Richness and Computacional Complexity. In: KAKAS, Antonis C.; FLACH, Peter (Ed.). Abduction and Induction: Essays on their Relation and Integration. Dordrecht: Kluwer, 2000, p. 59-74.

RIVADULLA, Andrés. Exito, Razón y Cambio en Física. Un enfoque instrumental en teoría de la ciencia. Madrid: Trotta, 2004.

SHELLEY, Cameron. Visual abductive reasoning in archaeology. Philosophy of Science, v. 53, p. 278-301, 1996.

SOAMES, Scott. What is Meaning? Princeton and Oxford: Princeton University Press, 2010.

SOAMES, Scott. Propositions. In: RUSSELL, Gillian; GRAFF, Delia (Ed.). Routledge Companion to Philosophy of Language. Routledge: Taylor \& Francis Group, p. 209-220, 2012.

SOAMES, Scott. Propositions as Cognitive Event Type. In: KING, Jeff; SOAMES, Scott; SPEAKS, Jeff (Ed.). New Thinking about Propositions. 2012 forthcoming (under contract with Oxford University Press).

STENINNG, Keith; LAMBALGEN, Michiel van. Semantics as a Foundation for Psychology: A Case Study of Wason's Selection Task. Journal of Logic, Language and Information, v. 10, p. 273-317, 2011.

THAGARD, Paul. The Best Explanation: Criteria for Theory Choice. The Journal of Philosophy, v. 75, p. 76-92, 1978.

THAGARD, Paul. Computacional Philosophy of Science. Cambridge, Mass: MIT Press., 1988.

TURSMAN, Richard. Peirce's Theory of Scientific Discovery: A System of Logic Conceived as Semiotics. Indianapolis: Indiana University Press, 1987.

Recebido em: 08.12.2012

Aceito em: 10.05.2012 